\title{
Exploiting direct link in EH based two-way DF half-duplex relaying network: Outage probability analysis
}

\author{
Phu Tran Tin", Minh Tran², Tran Thanh Trang ${ }^{3}$ \\ ${ }^{1}$ Faculty of Electronics Technology, Industrial University of Ho Chi Minh City, Ho Chi Minh City, Vietnam \\ ${ }^{2}$ Optoelectronics Research Group, Faculty of Electrical and Electronics Engineering, Ton Duc Thang University, \\ Ho Chi Minh City, Vietnam \\ ${ }^{3}$ National Key Laboratory of Digital Control and System Engineering, Ho Chi Minh City, Vietnam
}

\begin{tabular}{|c|c|}
\hline Article Info & ABSTRACT \\
\hline Article history: & Relay communication is considered as a popular solution for expanding \\
\hline Received Jun 4, 2019 & $\begin{array}{l}\text { the coverage, increasing the transmission capacity and reducing the power } \\
\text { consumption of the communication networks. In this paper we proposed }\end{array}$ \\
\hline Revised Sep 26, 2019 & and investigated the two-way decode-and-forward (DF) half-duplex (HD) \\
\hline Accepted Nov 30, 2019 & $\begin{array}{l}\text { relaying network with the direct link between two sources } S_{1} \text { and } S_{2} \text {. } \\
\text { Firstly, the system model, energy harvesting (EH) and information }\end{array}$ \\
\hline Keywords: & $\begin{array}{l}\text { transmission (IT) are presented. The closed-torm analytical expression } \\
\text { of the system outage probability (OP) is analyzed and derived in the next stage. }\end{array}$ \\
\hline Energy harvesting & Finally, the correctness of the analytical expressions is verified by Monte Carlo \\
\hline Half-duplex & simulation. The research results show that the analytical and simulation \\
\hline Monte Carlo & are the same in connection with the primary system parameters. \\
\hline Outage probability & \\
\hline Relaying network & This is an open access article under the $\underline{C C B Y-S A}$ license. \\
\hline \multicolumn{2}{|l|}{ Corresponding Author: } \\
\hline \\
\hline \multicolumn{2}{|c|}{ Optoelectronics Research Group, } \\
\hline \multicolumn{2}{|c|}{ Faculty of Electrical and Electronics Engineering, } \\
\hline \multicolumn{2}{|c|}{ Ton Duc Thang University, Ho Chi Minh City, Vietnam. } \\
\hline \multicolumn{2}{|c|}{ Email: tranhoangquangminh@tdtu.edu.vn } \\
\hline
\end{tabular}

\section{INTRODUCTION}

Nowadays, the fifth-generation $(5 \mathrm{G})$ wireless networks is considered as a hot research area in academic and industrial based on the increasing data rate transmission. Relay communication, in which the relay forwards the signal received by a source to a destination, has a massive consideration in research, due to its ability to expand the coverage, increase the capacity, and reduce the power consumption [1-4]. Moreover, relaying the communication network is an effective way to combat the performance degradation caused by fading, shadowing, path loss, and is an efficient way to improve spectrum efficiency and extend coverage [1-6]. On another way, two-way relaying, where two users exchange information with each other via a single or multiple relays, provides improved spectral efficiency compared to conventional one-way relaying by using either superposition coding or physical layer network coding at relays. Furthermore, to satisfy the 5G requirements, relaying schemes with high spectrum efficiency, such as two-way, and full-duplex, etc., have been recently attracted considerable attention [7-10]. In [7], the authors investigate one-way full-duplex (FD) relaying and two-way half-duplex (HD) relaying to minimize the spectral efficiency loss associated with one-way HD relaying. In [8] and [9], the authors proposed and investigated one-way FD relaying with multiple antennas to provide a solution to overcome the spectral efficiency loss. In [10], the authors investigate one-way FD relaying with opportunistic relay selection to enhance the performance. 
In this research, the two-way decode-and-forward (DF) HD relaying network with the direct link between two sources $S_{1}$ and $S_{2}$ is proposed and investigated. The system model, energy harvesting $(\mathrm{EH})$ and information transmission (IT) are presented in the first stage. After that, we derived the closed-form of the system outage probability (OP) in the next stage. Finally, the correctness of the analytical expressions is verified by Monte Carlo simulation in connection with the primary system parameters. The research results show that the analytical and simulation are the same in connection with the primary system parameters. In this research, we focus on some problems:

a. The two-way DF HD relaying network with the direct link between two sources $S_{1}$ and $S_{2}$ is proposed and investigated.

b. The closed-form expression of the system OP is analyzed and derived.

c. All the results are convinced by Monte Carlo simulation in connection with all primary system parameters.

The structure of the rest of the paper can be drawn as follows. We provide the system model, the energy and information transfer phases in section 2. The closed-form expression of system OP analysis is derived in section 3. We introduce the results and have some discussions in section 4. In the last section, some conclusions are proposed.

\section{RESEARCH METHOD}

In this section, the two-way DF HD relaying network with the direct link between two sources $S_{1}$ and $\mathrm{S}_{2}$ is drawn in Figure 1. The energy harvesting (EH) and information transformation (IT) for this proposed model system are proposed in Figure 2. In this protocol, the transmission interval time is $\mathrm{T}$, which consists of three-time slots $\mathrm{T} / 3$. In the first time slot $\mathrm{T} / 3$, the $\mathrm{R}$ harvests energy $\rho \mathrm{P}_{1}$ from the source node $\mathrm{S}_{1}$, and the source uses the energy $(1-\rho) \mathrm{P}_{1}$ for information transmission to $\mathrm{R}$ and $\mathrm{S}_{2}$ (here $0<\rho<1$ is the power splitting factor). In the second interval time $\mathrm{T} / 3$, the $\mathrm{R}$ harvests energy $\rho \mathrm{P}_{2}$ from the source node $S_{2}$, and the source $S_{2}$ uses the energy $(1-\rho) P_{2}$ for information transmission to $R$ and $S_{1}$. Finally, the remaining time slot $T / 3$ is used for information transferring from the $R$ to the nodes $S_{1}$ and $S_{2}$ [11-16].

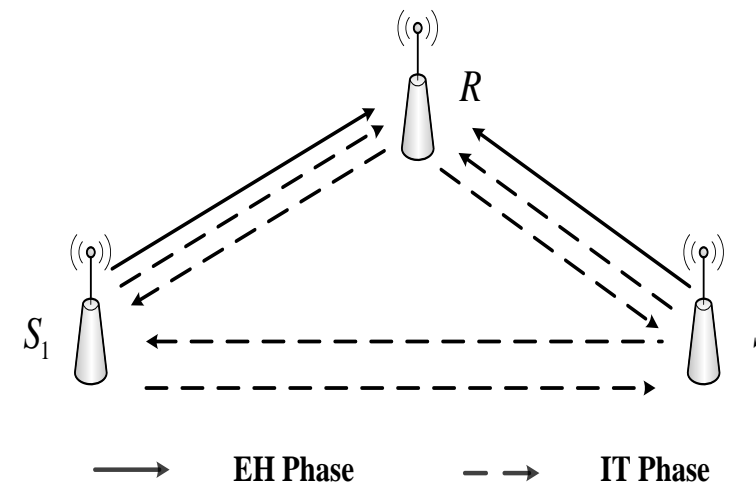

Figure 1. System model

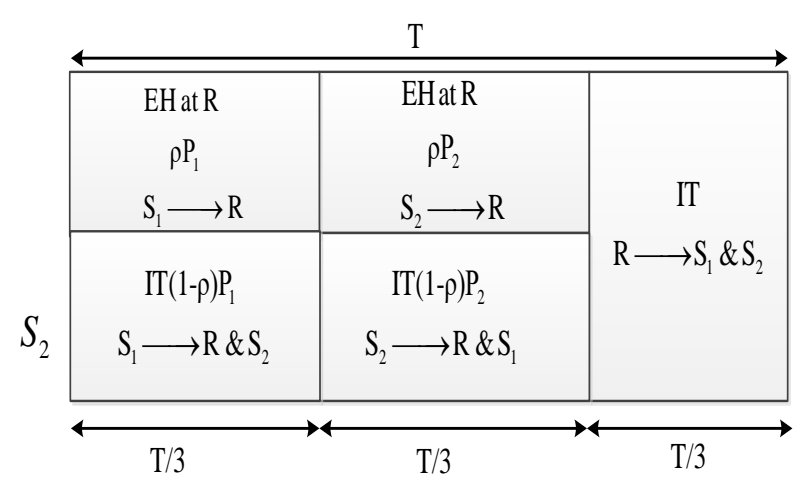

Figure 2. The energy harvesting $(\mathrm{EH})$ and information transmission (IT) processes

\subsection{Energy harvesting phase}

Let $S_{1}$ transmits the symbol $x_{1}$ in the first interval time. We define the received signal at the $\mathrm{R}$ node $\mathrm{R}$ and the $\mathrm{S}$ node $\mathrm{S}_{2}$ as:

$$
\begin{aligned}
& y_{1, R}^{I}=h_{1, R} x_{1}+n_{r}^{I} \\
& y_{1,2}^{I}=h_{1,2} x_{1}+n_{2}^{I}
\end{aligned}
$$

Where $\mathrm{E}\left\{\left|x_{1}\right|^{2}\right\}=P_{1}, \mathrm{E}\{\bullet\}$ is expectation operator and $\mathrm{P}_{1}$ represents the average transmit power at the $\mathrm{S}_{1}$. Further, $n_{r}^{I}$ and $n_{2}^{I}$ denote the zero-mean additive white Gaussian noise (AWGN) with variance $\mathrm{N}_{0}$. Then, the harvested energy at the $\mathrm{R}$ node can be formulated as the following: 


$$
E_{h}^{I}=\frac{\eta \rho P_{1}\left|h_{1, R}\right|^{2} T}{3}
$$

In the second interval time, the source node $S_{2}$ transmits the signal $x_{2}$ to the nodes $R$ and $S_{1}$. Therefore, the received signals at the $\mathrm{R}$ and $\mathrm{S}_{1}$ can be expressed, respectively, as:

$$
\begin{aligned}
& y_{2, R}^{I I}=h_{2, R} x_{2}+n_{r}^{I I}, \\
& y_{2,1}^{I I}=h_{2,1} x_{2}+n_{1}^{I I}
\end{aligned}
$$

Where $\mathrm{E}\left\{\left|x_{2}\right|^{2}\right\}=P_{2}, \mathrm{P}_{2}$ is the average transmit power at the $\mathrm{S}_{2}$. And we assume that $n_{r}^{I I}$ and $n_{1}^{I I}$ are the zero-mean additive white Gaussian noise (AWGN) with variance $\mathrm{N}_{0}$. Here, the total harvested energy at the $\mathrm{R}$ node can be defined as:

$$
E_{h}=\frac{\eta \rho\left(P_{1}\left|h_{1, R}\right|^{2}+P_{2}\left|h_{2, R}\right|^{2}\right) T}{3}
$$

In this model, the average transmits power from source $S_{1}$ and $S_{2}$ is assumed to equal. So, the (4) can be rewritten as the following:

$$
E_{h}=\frac{\eta \rho\left(P_{1}\left|h_{1, R}\right|^{2}+P_{2}\left|h_{2, R}\right|^{2}\right) T}{3}=\frac{\eta \rho P\left(\left|h_{1, R}\right|^{2}+\left|h_{2, R}\right|^{2}\right) T}{3}
$$

Where we denote $P_{1}=P_{2}=P$. Therefore, we have the average transmit power at the $\mathrm{R}$ as:

$$
P_{R}=\frac{E_{h}}{(T / 3)}=\eta \rho P\left(\left|h_{1, R}\right|^{2}+\left|h_{2, R}\right|^{2}\right)
$$

\subsection{Information transmission phase}

In the first interval time, after doing $\mathrm{EH}, \mathrm{S}_{1}$ broadcasts the information to the $\mathrm{R}$ node and $\mathrm{S}_{2}$ with remaining power $(1-\rho) P$. From that, we have the received signal at the R node and $\mathrm{S}_{2}$ as:

$$
\begin{aligned}
& y_{1, R}^{I}=\sqrt{1-\rho} h_{1, R} x_{1}+n_{r}^{I}, \\
& y_{1,2}^{I}=h_{1,2} x_{1}+n_{2}^{I}
\end{aligned}
$$

Similar to the first case, the received signal at node and $\mathrm{S}_{1}$ node can be given as the following:

$$
\begin{aligned}
& y_{2, R}^{I I}=\sqrt{1-\rho} h_{2, R} x_{2}+n_{r}^{I I}, \\
& y_{2,1}^{I I}=h_{2,1} x_{2}+n_{1}^{I I}
\end{aligned}
$$

Finally, in the third interval time, the received signal at the sources $S_{1}$ and $S_{2}$ can be expressed, respectively, as:

$$
\begin{aligned}
& y_{1}^{I I I}=h_{R, 1} x_{R}+n_{1}^{I I I}, \\
& y_{2}^{I I I}=h_{R, 2} x_{R}+n_{2}^{I I I}
\end{aligned}
$$

Where $\mathrm{E}\left\{\left|x_{R}\right|^{2}\right\}=P_{R}$. The received signal at the source node $\mathrm{S}_{1}$ or $\mathrm{S}_{2}$ can be formulated as:

$$
y_{1}^{D F}=\sqrt{P_{R}} h_{R, i}\left(x_{1} \oplus x_{2}\right)+n \quad y_{1}^{D F}=\sqrt{P_{R}} h_{R, i}\left(x_{1} \oplus x_{2}\right)+n \quad y_{1}^{D F}=\sqrt{P_{R}} h_{R, i}\left(x_{1} \oplus x_{2}\right)+n_{1}
$$


Where $i \in(1,2)$. Once again, after applying the canceling the self-interference method and combine with (6) the $\mathrm{SNR}$ at $\mathrm{S}_{1}$ can be defined as:

$$
\gamma_{1}^{D F}=\frac{P_{R}\left|h_{R, 1}\right|^{2}}{N_{0}}=\eta \rho \psi\left(\left|h_{1, R}\right|^{2}+\left|h_{2, R}\right|^{2}\right)\left|h_{R, 1}\right|^{2}=\eta \rho \psi \varphi_{1}\left[\varphi_{2}+\varphi_{4}\right]
$$

Where $\varphi_{4}=\left|h_{1, R}\right|^{2}$

\section{SYSTEM PERFORMANCE ANALYSIS}

Hence, the outage probability (OP) of DF mode at the $S_{1}$ can be formulated as:

$$
\begin{aligned}
O P_{D F}= & \operatorname{Pr}\left\{\gamma_{c 2 e}^{D F}<\gamma_{t h}\right\}=\operatorname{Pr}\left\{\max \left\{\eta \rho \psi \varphi_{1}\left[\varphi_{2}+\varphi_{4}\right], \psi \varphi_{3}\right\}<\gamma_{t h}\right\} \\
& =\operatorname{Pr}\left\{\eta \rho \varphi \varphi_{1}\left[\varphi_{2}+\varphi_{4}\right]<\gamma_{t h}\right\} \times \operatorname{Pr}\left\{\psi \varphi_{3}<\gamma_{t h}\right\}
\end{aligned}
$$

Where we denote:

$$
\operatorname{Pr}\left\{\psi \varphi_{3}<\gamma_{t h}\right\}=\operatorname{Pr}\left\{\varphi_{3}<\frac{\gamma}{\psi}\right\}=1-\exp \left(-\frac{\gamma_{t h}}{\lambda_{3} \psi}\right)
$$

Which $\lambda_{3}$ is mean of the random variable (RV) $\varphi_{3}$. Here we have:

$$
\begin{aligned}
P_{3} & =\operatorname{Pr}\left\{\eta \rho \psi \varphi_{1} \varphi<\gamma_{t h}\right\}=\operatorname{Pr}\left\{\varphi_{1}<\frac{\gamma_{t h}}{\eta \rho \psi \varphi}\right\} \\
& =\int_{0}^{\infty} F_{\varphi_{1}}\left(\frac{\gamma_{t h}}{\eta \rho \psi \varphi} \mid \varphi\right) f_{\varphi}(\varphi) d \varphi
\end{aligned}
$$

Where $\varphi=\varphi_{2}+\varphi_{4}$ For calculating the probability in (13), the probability density function $(\mathrm{PDF})$ and cumulative density function (CDF) of $\varphi$ is calculated as in Lemma 1

Lemma 1.

The CDF of $\left(\varphi_{2}+\varphi_{4}\right)$ can be formulated as:

$$
\begin{aligned}
F_{\varphi}(x)=\operatorname{Pr} & {[\varphi<x]=\operatorname{Pr}\left[\varphi_{2}<x-\varphi_{4}\right] } \\
& =\int_{0}^{x} f_{\varphi_{4}}\left(\varphi_{4}\right) d \varphi_{4} \int_{0}^{x-\varphi_{4}} f_{\varphi_{2}}\left(\varphi_{2}\right) d \varphi_{2}=\int_{0}^{x} F_{\varphi_{2}}\left(x-\varphi_{4}\right) f_{\varphi_{4}}\left(\varphi_{4}\right) d \varphi_{4} \\
& =\frac{1}{\lambda_{4}} \int_{0}^{x}\left[1-\exp \left(-\frac{x-\varphi_{4}}{\lambda_{2}}\right)\right] \exp \left(-\frac{\varphi_{4}}{\lambda_{4}}\right) d \varphi_{4} \\
& =1-\exp \left(-\frac{x}{\lambda_{4}}\right)-\frac{\exp \left(-\frac{x}{\lambda_{2}}\right)}{\lambda_{4}} \int_{0}^{x} \exp \left(\varphi_{4}\left[\frac{1}{\lambda_{2}}-\frac{1}{\lambda_{4}}\right]\right) d \varphi_{4}
\end{aligned}
$$

Where $\lambda_{4}$ is the mean of $\mathrm{RV} \varphi_{4}$.

Case 1: $\lambda_{2}=\lambda_{4}=\lambda$

From (28), we have:

$$
F_{\varphi}(x)=1-\exp \left(-\frac{x}{\lambda}\right)-\frac{x \exp \left(-\frac{x}{\lambda}\right)}{\lambda}
$$


And the PDF of $\varphi$ can be computed as:

$$
f_{\varphi}(x)=\frac{\partial F_{\varphi}(x)}{\partial x}=\frac{x \exp \left(-\frac{x}{\lambda}\right)}{\lambda^{2}}
$$

Case 2: $\lambda_{2} \neq \lambda_{4}$

Similar, we can obtain CDF and PDF of $\varphi$ as followings respectively:

$$
\begin{aligned}
& F_{\varphi}(x)=1-\exp \left(-\frac{x}{\lambda_{4}}\right)-\frac{\lambda_{2}}{\lambda_{4}-\lambda_{2}}\left[\exp \left(-\frac{x}{\lambda_{4}}\right)-\exp \left(-\frac{x}{\lambda_{2}}\right)\right] \\
& f_{\varphi}(x)=\frac{1}{\lambda_{4}-\lambda_{2}}\left[\exp \left(-\frac{x}{\lambda_{4}}\right)-\exp \left(-\frac{x}{\lambda_{2}}\right)\right]
\end{aligned}
$$

We can calculate the probability $\mathrm{P}_{3}$ into 2 case

Case 1: $\lambda_{2}=\lambda_{4}=\lambda$

Substituting (14) into (16), we can obtain:

$$
\begin{aligned}
P_{3} & =1-\int_{0}^{\infty} \exp \left[-\frac{\gamma_{t h}}{\eta \rho \psi \varphi \lambda_{1}}\right] \times \frac{\varphi \exp \left(-\frac{\varphi}{\lambda}\right)}{\lambda^{2}} d \varphi \\
& =1-\frac{1}{\lambda^{2}} \int_{0}^{\infty} \varphi \times \exp \left[-\frac{\gamma_{t h}}{\eta \rho \psi \varphi \lambda_{1}}\right] \times \exp \left(-\frac{\varphi}{\lambda}\right) d \varphi
\end{aligned}
$$

Apply $(3.471,9)$ in [17], (20) can be rewritten as:

$$
P_{3}=1-\frac{2 \gamma_{t h}}{\eta \rho \psi \lambda \lambda_{1}} K_{2}\left(2 \sqrt{\frac{\gamma_{t h}}{\eta \rho \psi \lambda \lambda_{1}}}\right)
$$

Where $K_{v}(\bullet)$ is the modified Bessel function of the second kind and $\mathrm{v}^{\text {th }}$ order.

Also, finally, substituting (13), (21) into (12), the system OP at the source $S_{1}$ in the first interval time can be obtained as:

$$
O P_{D F}^{1}=\left\{1-\exp \left(-\frac{\gamma_{t h}}{\lambda_{3} \psi}\right)\right\}\left\{1-\frac{2 \gamma_{t h}}{\eta \rho \psi \lambda \lambda_{1}} K_{2}\left(2 \sqrt{\frac{\gamma_{t h}}{\eta \rho \psi \lambda \lambda_{1}}}\right)\right\}
$$

Case 2: $\lambda_{2} \neq \lambda_{4}$

Substituting (19) into (14), the probability $\mathrm{P}_{3}$ can be expressed as:

$$
\begin{aligned}
P_{3} & =1-\int_{0}^{\infty} \exp \left[-\frac{\gamma_{t h}}{\eta \rho \psi \varphi \lambda_{1}}\right] \times \frac{1}{\lambda_{4}-\lambda_{2}}\left[\exp \left(-\frac{\varphi}{\lambda_{4}}\right)-\exp \left(-\frac{\varphi}{\lambda_{2}}\right)\right] d \varphi \\
& =1-\frac{1}{\lambda_{4}-\lambda_{2}} \int_{0}^{\infty} \exp \left[-\frac{\gamma_{t h}}{\eta \rho \psi \varphi \lambda_{1}}\right] \times\left[\exp \left(-\frac{\varphi}{\lambda_{4}}\right)-\exp \left(-\frac{\varphi}{\lambda_{2}}\right)\right] d \varphi \\
& =1-\frac{1}{\lambda_{4}-\lambda_{2}} \int_{0}^{\infty} \exp \left[-\frac{\gamma_{t h}}{\eta \rho \psi \varphi \lambda_{1}}\right] \times \exp \left(-\frac{\varphi}{\lambda_{4}}\right) d \varphi \\
& +\frac{1}{\lambda_{4}-\lambda_{2}} \int_{0}^{\infty} \exp \left[-\frac{\gamma_{t h}}{\eta \rho \psi \varphi \lambda_{1}}\right] \times \exp \left(-\frac{\varphi}{\lambda_{2}}\right) d \varphi
\end{aligned}
$$

Apply eq $(3.324,1)$ in [17], (23) can be rewritten as: 


$$
P_{3}=1-\frac{2}{\lambda_{4}-\lambda_{2}} \sqrt{\frac{\gamma_{t h}}{\eta \rho \psi \lambda_{1}}} \times\left\{\begin{array}{l}
\sqrt{\lambda_{4}} \times K_{1}\left(2 \sqrt{\frac{\gamma_{t h}}{\eta \rho \psi \lambda_{1} \lambda_{4}}}\right) \\
-\sqrt{\lambda_{2}} \times K_{1}\left(2 \sqrt{\frac{\gamma_{t h}}{\eta \rho \psi \lambda_{1} \lambda_{2}}}\right)
\end{array}\right\}
$$

And then substituting (13), (24) into (12), the OP at the source $S_{1}$ in the second case can be obtained as:

$$
O P_{D F}^{2}=\left[1-\exp \left(-\frac{\gamma_{t h}}{\lambda_{3} \psi}\right)\right]\left[1-\frac{2}{\lambda_{4}-\lambda_{2}} \sqrt{\frac{\gamma_{t h}}{\eta \rho \psi \lambda_{1}}} \times\left\{\begin{array}{l}
\sqrt{\lambda_{4}} \times K_{1}\left(2 \sqrt{\frac{\gamma_{t h}}{\eta \rho \psi \lambda_{1} \lambda_{4}}}\right) \\
-\sqrt{\lambda_{2}} \times K_{1}\left(2 \sqrt{\frac{\gamma_{t h}}{\eta \rho \psi \lambda_{1} \lambda_{2}}}\right)
\end{array}\right\}\right]
$$

\section{NUMERICAL RESULTS AND DISCUSSION}

In this section, we investigate the system OP using Monte Carlo simulation in connection with the primary system parameters [18-25]. In Figure 3, the effect of the energy coefficient $\eta$ on the system OP is plotted with the primary system parameters as $\rho=0.8, \mathrm{R}=0.5 \mathrm{bps} / \mathrm{Hz}, \psi=0.5 \mathrm{~dB}$. From the results, we can see that the OP of $S_{1}$ significantly decreases with the rising of $\eta$. Here, we consider both cases with and without a direct link between the sources $S_{1}$ and $S_{2}$. From Figure 3, we can see that the system OP with the direct link is better than the system OP without the direct link between the sources. Furthermore, the simulation results agree well with the analytical results. In the same way, the system OP versus $\psi$ is drawn in Figure 4. In this simulation, we set $\eta=0.5, \mathrm{R}=0.5 \mathrm{bps} / \mathrm{Hz}, \rho=0.5$, and consider both cases with and without a direct link between the sources. As shown in Figure 4, we can state that the system OP has a considerable decrease when $\psi$ varies from-10 to 10 . In Figure 4, the simulation and analytical results are the same with all values of $\alpha$ and $b_{2}$, and again, the OP with the direct link is better than the cases without.

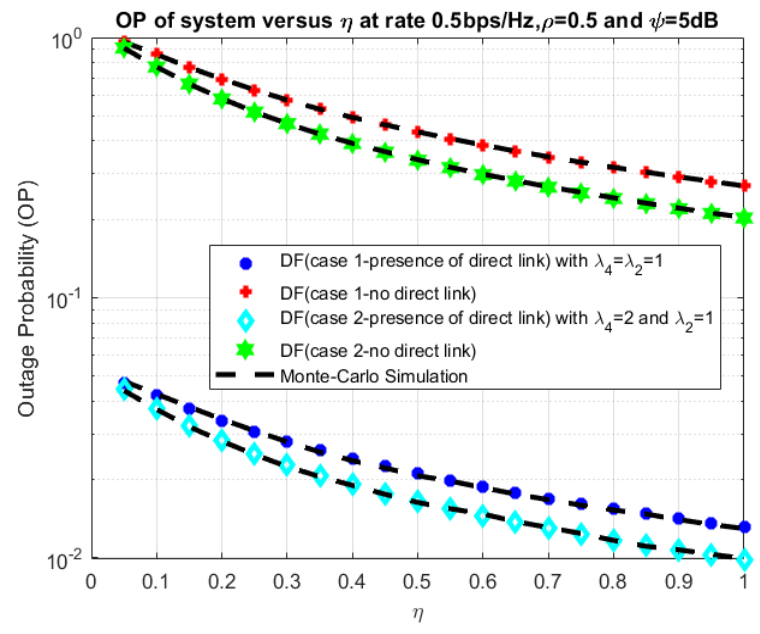

Figure 3. OP versus $\eta$

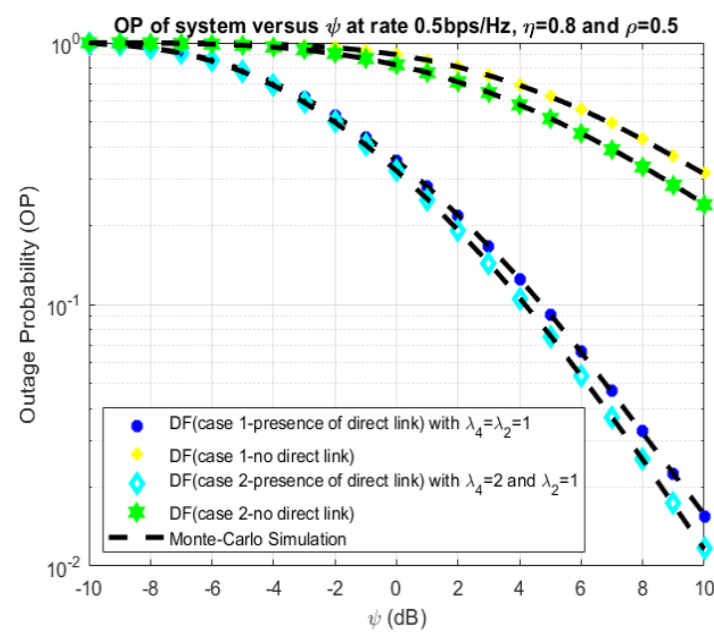

Figure 4. OP versus $\psi$

Furthermore, we investigate the effect of $\mathrm{R}$ and $\rho$ on the OP of the model system, as plotted in Figures 5 and 6. In these Figures, we set the primary parameters, as shown in these Figures. From Figures 5 and 6 , it can be observed that OP has a slight decrease when $\rho$ varies from 0 to 1 , and the system OP crucially increases with the rising of $\mathrm{R}$ from 0.5 to 5 . In all two Figures, the analytical and simulation results agree well with each other. On another hand, the comparison of the system OP of both cases with and without a direct link between these sources is demonstrated in all Figures. From the results, we can state that the system OP with the direct link is better than another case. 


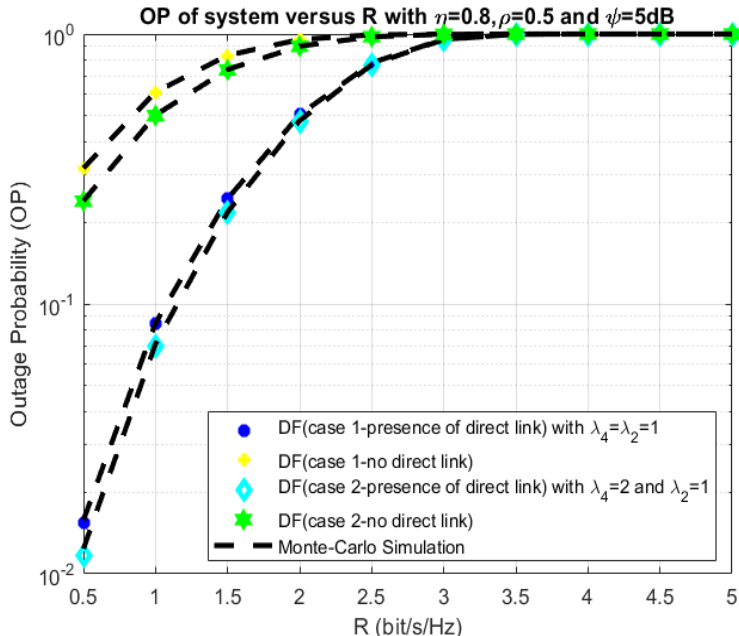

Figure 5. OP versus R

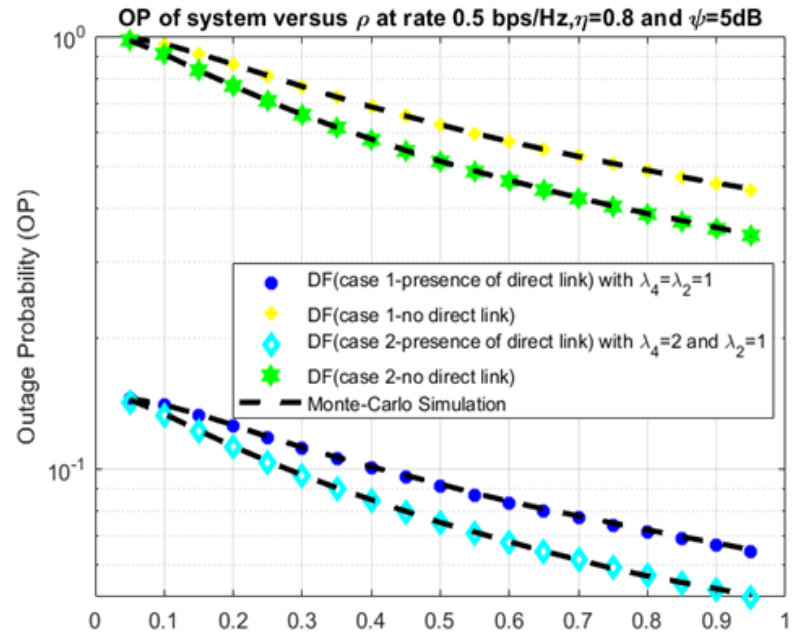

Figure 6. OP versus $\rho$

\section{CONCLUSION}

In this research, the two-way DF HD relaying network with the direct link between two sources $\mathrm{S}_{1}$ and $S_{2}$ is proposed and investigated. The system model, EH and IT are presented in the first stage. After that, we derived the closed-form expression of the system OP in the next stage. Finally, the correctness of the analytical expressions is verified by Monte Carlo simulation in connection with the primary system parameters. The research results show that the analytical and simulation are the same as the primary system parameters. The results can provide a critical recommendation for the relaying communication network.

\section{ACKNOWLEDGEMENTS}

This research was supported by National Key Laboratory of Digital Control and System Engineering (DCSELAB), HCMUT, VNU-HCM, Vietnam.

\section{REFERENCES}

[1] S. Bi, C. K. Ho and R. Zhang, "Wireless powered communication: opportunities and challenges," in IEEE Communications Magazine, vol. 53, no. 4, pp. 117-125, April 2015.

[2] D. Niyato, D. I. Kim, M. Maso and Z. Han, "Wireless Powered Communication Networks: Research Directions and Technological Approaches," in IEEE Wireless Communications, vol. 24, no. 6, pp. 88-97, Dec. 2017.

[3] H. Yu, H. Lee, \& H. Jeon, "What is 5G? Emerging 5G Mobile Services and Network Requirement," Sustainability, vol. 9, no. 10, pp. 1848, 2017.

[4] M. Duarte, C. Dick and A. Sabharwal, "Experiment-Driven Characterization of Full-Duplex Wireless Systems," in IEEE Transactions on Wireless Communications, vol. 11, no. 12, pp. 4296-4307, December 2012.

[5] W. C. Y. Lee, "The most spectrum-efficient duplexing system: CDD," in IEEE Communications Magazine, vol. 40, no. 3, pp. 163-166, March 2002.

[6] C. Wang et al., "Cellular architecture and key technologies for 5G wireless communication networks," in IEEE Communications Magazine, vol. 52, no. 2, pp. 122-130, February 2014.

[7] H. Ju, E. Oh and D. Hong, "Catching resource-devouring worms in next-generation wireless relay systems: Two-way relay and full-duplex relay," in IEEE Communications Magazine, vol. 47, no. 9, pp. 58-65, September 2009

[8] T. Riihonen, S. Werner, R. Wichman and J. Hamalainen, "Outage Probabilities in Infrastructure-Based Single-Frequency Relay Links," 2009 IEEE Wireless Communications and Networking Conference, Budapest, pp. $1-6,2009$.

[9] D. W. K. Ng, E. S. Lo and R. Schober, "Dynamic Resource Allocation in MIMO-OFDMA Systems with Full-Duplex and Hybrid Relaying," in IEEE Transactions on Communications, vol. 60, no. 5, pp. 1291-1304, May 2012.

[10] I. Krikidis, H. A. Suraweera, P. J. Smith and C. Yuen, "Full-Duplex Relay Selection for Amplify-and-Forward Cooperative Networks," in IEEE Transactions on Wireless Communications, vol. 11, no. 12, pp. 4381-4393, December 2012. 
[11] S. Luo, R. Zhang and T. J. Lim, "Optimal save-then-transmit protocol for energy harvesting wireless transmitters," 2012 IEEE International Symposium on Information Theory Proceedings, Cambridge, MA, pp. 955-959, 2012.

[12] Tan N. Nguyen, T. H. Q. Minh, Phuong T. Tran and Miroslav Voznak, "Energy Harvesting over Rician Fading Channel: A Performance Analysis for Half-Duplex Bidirectional Sensor Networks under Hardware Impairments," Sensors, vol. 18, no. 6, pp. 1781, 2018.

[13] Tan N. Nguyen, T. H. Q. Minh, Phuong T. Tran and Miroslav Voznak, "Adaptive Energy Harvesting Relaying Protocol for Two-Way Half Duplex System Network over Rician Fading Channel," Wireless Communications and Mobile Computing, 2018.

[14] H. A. Suraweera, G. K. Karagiannidis and P. J. Smith, "Performance analysis of the dual-hop asymmetric fading channel," in IEEE Transactions on Wireless Communications, vol. 8, no. 6, pp. 2783-2788, June 2009.

[15] M. R. Bhatnagar, "On the Capacity of Decode-and-Forward Relaying over Rician Fading Channels," in IEEE Communications Letters, vol. 17, no. 6, pp. 1100-1103, June 2013.

[16] Tan N. Nguyen, T. H. Q. Minh, Phuong T. Tran, Miroslav Voznak, T. T. Duy, Thanh-Long Nguyen and Phu Tran Tin, "Performance Enhancement for Energy Harvesting Based Two-Way Relay Protocols in Wireless Ad-hoc Networks with Partial and Full Relay Selection Methods," Ad hoc Networks, Vol.84, pp. 178-187, Mar. 2019.

[17] Table of Integrals, Series, and Products, 2015.

[18] C. R. Valenta and G. D. Durgin, "Harvesting Wireless Power: Survey of Energy-Harvester Conversion Efficiency in Far-Field, Wireless Power Transfer Systems," in IEEE Microwave Magazine, vol. 15, no. 4, pp. 108-120, June 2014.

[19] R. H. Y. Louie, Y. Li and B. Vucetic, "Practical physical layer network coding for two-way relay channels: performance analysis and comparison," in IEEE Transactions on Wireless Communications, vol. 9, no. 2, pp. 764-777, February 2010.

[20] T. Riihonen, S. Werner and R. Wichman, "Hybrid Full-Duplex/Half-Duplex Relaying with Transmit Power Adaptation," in IEEE Transactions on Wireless Communications, vol. 10, no. 9, pp. 3074-3085, September 2011.

[21] Y. Deng, L. Wang, M. Elkashlan, K. J. Kim and T. Q. Duong, "Generalized Selection Combining for Cognitive Relay Networks Over Nakagami-m Fading," in IEEE Transactions on Signal Processing, vol. 63, no. 8, pp. 1993-2006, April15, 2015.

[22] Tin, Phu Tran, Tran Hoang Quang Minh, Tan N. Nguyen, and Miroslav Voznak, "System Performance Analysis of Half-Duplex Relay Network over Rician Fading Channel," TELKOMNIKA (Telecommunication, Computing, Electronics and Control), vol. 16, no. 1, pp. 189-199, 2018.

[23] Rashid, Tarique, Sunil Kumar, Akshay Verma, Prateek Raj Gautam, and Arvind Kumar, "Pm-EEMRP: Postural Movement Based Energy Efficient Multi-hop Routing Protocol for Intra Wireless Body Sensor Network (Intra-WBSN)," TELKOMNIKA (Telecommunication, Computing, Electronics and Control), vol. 16, no. 1, pp. 166-173, 2018.

[24] A. F. Morabito, "Power Synthesis of Mask-Constrained Shaped Beams Through Maximally-Sparse Planar Arrays," TELKOMNIKA (Telecommunication Computing Electronics and Control), vol. 14, no. 4, pp. 1217-1219, 2016.

[25] C. Wang, J. Li, Y. Yang and F. Ye, "Combining Solar Energy Harvesting with Wireless Charging for Hybrid Wireless Sensor Networks," in IEEE Transactions on Mobile Computing, vol. 17, no. 3, pp. 560-576, 1 March 2018. 